\title{
LE PROBLEME DES ERREURS DU POINT DE VUE DE \\ L'OBSERVATEUR ET DU POINT DE VUE DU \\ CALCULATEUR D'ORBITES
}

\author{
S. AREND
}

Observatoire Royal de Belgique, Bruxelles, Belgique

\begin{abstract}
Resumé. En principe, nous parlerons des mesures d'étoiles doubles exécutées au micromètre à fils par la méthode classique des doubles distances, mais il va de soi que la plupart des considérations relatives aux erreurs peuvent être étendues aux autres procédés.
\end{abstract}

\section{Présentation des résultats d'observation}

Les moyennes conclues sont en général des moyennes arithmétiques simples. Dans certains cas, il s'agit de moyennes pondérées où les poids sont attribués, par exemple, d'après la correspondance suivante:

\begin{tabular}{llll} 
État des images: & 1 & 2 & 3 \\
& Très bon & Bon & Passable \\
Poids & 3 & 2 & 1 \\
\hline
\end{tabular}

Lorsqu'on considère les publications des auteurs, on constate que ceux-ci ont procédé de quatre façons: (a) les observations isolées sont mentionnées sans formation des moyennes; (b) les moyennes arithmétiques sont publiées pour les $t, \theta$ et $\varrho$; (c) les moyennes pondérées sont déterminées pour les $t, \theta$ et $\varrho$; (d) les moyennes arithmétiques sont données pour les dates tandis que les moyennes pondérées sont calculées pour les $\theta$ ainsi que pour les $\varrho$.

Parfois des moyennes générales sont calculées en pondérant les résultats des observations d'après le nombre de nuits.

Aucune remarque n'est à formuler au sujet des trois premières façons de procéder (a), (b) et (c). Mais que penser de la quatrième façon (d) à laquelle l'observateur peut être enclin de recourir en se disant que les temps étant exacts il n'y a pas lieu de les pondérer tandis qu'au contraire les coordonnées polaires $\theta$ et $\varrho$ sont à pondérer puisqu'elles ont une qualité relevant des poids.

Nous nous proposons de montrer sur un exemple (avec poids sciemment forcés pour les besoins de l'illustration) que cette dernière façon est incorrecte.

Imaginons les observations suivantes, que nous supposons exactes:

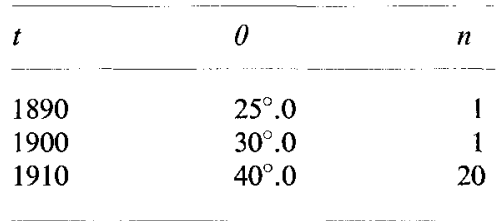


Formons les moyennes pour les deux dernières observations comme prévu en (b), (c) et (d):

(b) Moyenne arithmétique pour les $t, \theta$ et $\varrho$ :

$$
\frac{1900+1910}{2}=1905.0 ; \quad \frac{30.0+40.0}{2}=35^{\circ} .0 \text {. }
$$

(c) Moyenne pondérée pour les $t, \theta$ et $\varrho$ :

$$
\frac{1900+20 \times 1900}{21}=1909.5 ; \quad \frac{30.0+20 \times 40^{\circ} .0}{21}=39^{\circ} .5 .
$$

(d) Moyenne arithmétique pour les $t$ et moyenne pondérée pour les $\theta$ et $\varrho$ :

$$
\frac{1900+1910}{2}=1905.0 ; \quad \frac{30.0+20 \times 40^{\circ} .0}{21}=39^{\circ} .5 \text {. }
$$

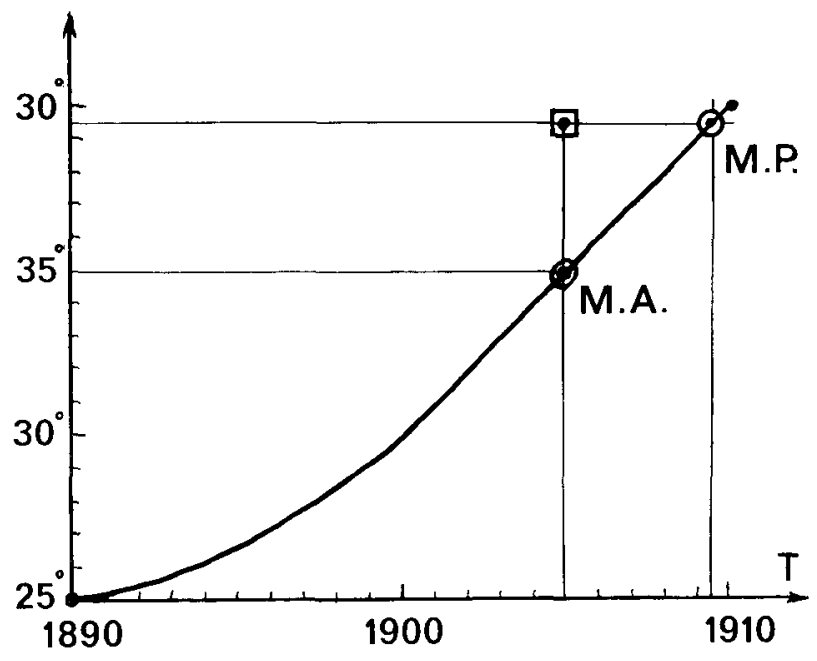

Fig. 1.

Ces résultats, portés en graphique, font ressortir que seules les façons de procéder (b) (moyenne arithmétique générale M.A.) et (c) (moyenne pondérée générale M.P.) sont à prendre en considération selon le jugement de l'observateur. La façon (d) de calculer est à rejeter. Cette assertion peut faire l'objet d'une démonstration rigoureuse. En effet, dans les domaines limités où on les considère, les $\theta$ (ou les $\varrho$ ) peuvent être envisagés comme étant liés aux $t$ par la relation linéaire

$$
\theta=a t+b
$$

où les $t$ sont exacts tandis que les $\theta$ sont entachés d'erreurs aléatoires. En conséquence, si lors du calcul des paramètres $a$ et $b$ par la méthode des moindres carrés on affecte les $\theta_{i}$ de poids $p_{i}$, cela implique que les $t_{t}$ sont aussi, en même temps, affectés des mêmes poids. 


\section{Précision des mesures}

Il n'y a pas lieu, pensons-nous, d'examiner cette question dans le détail, vu que chaque observateur la connaît bien puisqu'il s'y réfère dans le but de caractériser la précision de son travail pour son information personnelle et pour celle des usagers.

Les appréciations figurent d'ailleurs bien souvent dans les introductions précédant la publication des mesures faites par les professionnels. Les mesures isolées, comparées à leurs moyennes respectives, permettent de fixer la valeur des erreurs moyennes $\varepsilon_{\theta}$ en angle de position et $\varepsilon_{\ell}$ en distance, en l'occurrence, en recourant à la formule rappelée tout à l'heure par J. Dommanget. Contentons-nous de mentionner les résultats obtenus pour caractériser, d'après les distances des composantes des couples, la valeur personnelle du travail que nous avons effectué à l'aide du réfracteur de $45 \mathrm{~cm}$ de l'Observatoire Royal de Belgique [1]:

\begin{tabular}{rrr}
\hline Distances & \multicolumn{1}{c}{$\varepsilon_{\theta}$} & $\varepsilon_{\rho}$ \\
$0^{\prime \prime} .00-0^{\prime \prime} .60$ & $\pm 1^{\circ} .3$ & $\pm 0^{\prime \prime} .05$ \\
$0.61-1.00$ & 1.2 & 0.04 \\
$1.01-2.00$ & 1.1 & 0.07 \\
$2.01-4.00$ & 1.0 & 0.07 \\
$4.01-8.00$ & 1.3 & 0.06 \\
8.01 & $(1.2)$ & $(0.05)$ \\
Moyennes & $\pm 1^{\circ} .2$ & $\pm 0^{\prime \prime} .06$
\end{tabular}

Les erreurs moyennes ci-dessus résultant exclusivement de la prise en considération des mesures effectuées par l'observateur, sont ce que l'on pourrait appeler les erreurs intrinsèques des observations faites par cet observateur.

La probabilité qu'une erreur dépasse 3 fois l'erreur moyenne étant de 0.999 , on peut admettre que la quantité $3 \times$ e.m. représente le maximum pratique susceptible d'être constaté entre le résultat d'une observation et sa valeur exacte. Cela signifie, si l'on s'en réfère au tableau ci-dessus, qu'en principe les écarts entre les moyennes conclues et les mesures isolées ont pu atteindre au plus des valeurs de l'ordre de \pm 3.6 et \pm 0 "18. Le praticien pourra faire application du critérium de Hayford: "Eliminer toute observation dont le résidu dépasse 3.5 e.m. et vérifier toute observation dont le résidu dépasse 2.5 e.m."

Comment parvenir à déterminer les erreurs extrinsèques des observations susceptibles de caractériser le degré de confiance que l'on peut avoir dans leur utilisation? Pour ce qui concerne nos propres observations faites à Uccle, nous avons considéré les résidus $O-C$ résultant de la comparaison des moyennes publiées sous la forme habituelle ( $t, \theta, \varrho$ et $n$, nombre de nuits) avec les éphémérides d'orbites existantes; parmi ces résidus, nous avons choisi ceux dont les variations pouvaient s'expliquer par les discordances entre nos diverses mesures et, en principe, non par une mauvaise qualité des orbites. Pour des couples rangés par ordre de $\varrho$ croissants, nous avons déterminé les écarts à la moyenne des résidus et calculé les erreurs moyennes quadratiques. Voici ces résultats: 


$\begin{array}{lllll}\varrho \text { moyen: } & 0^{\prime \prime} .60 & 1^{\prime \prime} .10 & 1^{\prime \prime} .60 & 5^{\prime \prime} .50 \\ & & & & \\ \varepsilon_{\theta} & \left\llcorner 1^{\circ} .9\right. & +1^{\circ} .7 & +1^{\circ} .5 & +0^{\circ} .9 \\ \varepsilon_{\ell} & \pm 0^{\prime \prime} .02 & \pm 0^{\prime \prime} .03 & +0^{\prime \prime} .04 & +0^{\prime \prime} .11\end{array}$

Dans la matière qui nous occupe, il faut bien en convenir: le calcul des probabilités ne peut fournir que des ordres de grandeur. Cependant, nous pensons que les deux tableaux numériques ci-dessus suffisent à caractériser le degré d'homogénéité des observations et qu'ils peuvent servir de guide à l'utilisateur qui s'occupe de l'exploitation de celles-ci.

Nous ne nous étendrons pas sur la question des erreurs systématiques comme celles qui se présentent dans les distances de couples serrés et dont certains auteurs corrigent leurs résultats avant de les publier. Nous pensons que cette façon de faire risque d'entraîner des confusions et qu'il vaudrait mieux voir les résultats publiés tels qu'ils ont été obtenus; en effet, le soin de leur correction peut être laissé à l'utilisateur qui se référera aux directives données par l'observateur même.

\section{Poids 'a priori'}

Les poids des observations, attribués a priori, relèvent généralement de l'empirisme. Pour les fixer, on peut tenir compte de la valeur de tel observateur selon sa réputation, son expérience, l'ouverture de l'instrument utilisé, etc. Si l'étoile double est observée à l'aide d'une lunette dont le pouvoir séparateur est suffisant pour la mesure convenable du couple, on pourra trouver légitime de ne prendre en considération que le nombre de nuits.

Ces poids ont d'ailleurs à intervenir de diverses façons selon les problèmes à résoudre.

Ainsi, lorsqu'il s'agit de tracer au mieux les courbes $\theta(t)$ et $\varrho(t)$, on peut tenir compte du poids des observations et appliquer le principe des moyennes pondérées pour marquer les points par lesquels on fera passer respectivement les dites courbes. Cette façon de prendre en considération des centres de distances proportionnelles, comme cela se fait parfois en astrographie [2], et non des centres de moyennes distances est quasi nécessaire lorsque la qualité des observations est disparate.

Par contre, lorsqu'il s'agit d'amélioration d'orbites, certains calculateurs font usage de poids réduits à utiliser dans la formation des lieux normaux de base en se fondant sur l'expérience révélant qu'une observation résultant de mesures faites durant 1 nuit ne mérite qu'un poids minime alors que des moyennes faites sur des observations relatives à 2 jusqu'à 6 nuits méritent à peu près le même poids. A titre d'exemple, on peut se référer à la façon de procéder suivie par Wierzbiński [3] pour améliorer l'orbite de ADS 8337, couple observé en 69 ans sur un arc de $342^{\circ}$ jusqu'en 1952, année du calcul de l'auteur. Les distances observées se situant entre 0.15 et 0.60, cet auteur se conforme à la règle suivante: les observations exécutées pendant une seule nuit ou effectuées à l'aide d'un instrument dont le diamètre est inférieur à 11 pouces ont reçu 
le poids $p=\frac{1}{2}$ et les moyennes de 2 à 6 nuits, le poids $p=1$. Quant à deux observations manifestement discordantes, elles furent omises ou, si l'on veut, elles reçurent le poids 0 . Cela permet à l'auteur de baser l'amélioration de l'orbite sur 14 lieux normaux dont les poids s'échelonnent entre 1 et 5 comme suit: $1,2,3,3 \frac{1}{2}, 4,4 \frac{1}{2}, 5$.

Tout ce qui vient d'être dit a trait à la formation et à l'utilisation de poids habituellement dénommés 'poids a priori'. La discussion autour de ces questions a déjà fait couler beaucoup d'encre, mais on peut sans doute reconnaître la légitimité des principes mis en cuvre et la valeur des travaux ainsi accomplis.

\section{Poids 'a posteriori'}

Les poids 'a posteriori' sont des poids obtenus par le calcul et dont se servent certains auteurs lors de la détermination de ce qu'ils appellent, peut-être un peu abusivement, l'orbite définitive d'une étoile double visuelle.

On sait que le problème des erreurs comporte une complication du fait que les observateurs ont leur équation personnelle, ce qui conduit à des erreurs systématiques; cependant, si l'on admet que celles-ci se rangent dans la catégorie Il de Gauss, c'est-àdire si elles présentent une grande variété en sorte que les lois du hasard leur soient applicables, on peut se poser la question de chercher à fixer les poids par le calcul.

Admettons que l'on dispose d'une orbite d'étoile double que l'on améliore éventuellement quelque peu en sorte que l'on puisse dire quelle représente d'une manière satisfaisante l'ensemble des observations, qui sont très nombreuses et qui proviennent de nombreux observateurs. Comme cette orbite doit être très voisine de l'orbite définitive, il semble légitime de pouvoir s'en servir pour fixer les poids $p_{i}$ des observateurs. C'est ce que fait en particulier Karmel [4] à qui nous nous permettrons de renvoyer les intéressés désireux de scruter cette question dans le détail. Contentons-nous de dire que cet auteur détermine d'abord ce qu'il appelle les erreurs provisoires d'observation, qu'il rassemble pour chaque observateur désigné, puis s'en sert pour calculer l'erreur moyenne $\eta$ de cet observateur. Désignant par $\eta_{1}, \eta_{2}, \ldots$ les erreurs moyennes des divers observateurs et, prenant comme observation étalon celle dont l'erreur moyenne est $\eta_{0}$ et le poids $p_{0}$, on a

$$
p_{1} \eta_{1}^{2}=p_{2} \eta_{2}^{2}=\cdots=p_{0} \eta_{0}^{2}
$$

pouvant servir à fixer les poids $p_{i}$ des observateurs ou plutôt à servir de guide dans la fixation de ces poids, car on sait qu'il est superflu de prendre ceux-ci trop élevés.

En effet, on n'ignore pas que si l'on compense entre elles des mesures absolument concordantes les poids respectifs qu'on juge pouvoir leur attribuer n'a pas d'influence sur le résultat; c'est ce qu'a notamment fait remarquer l'astronome français Volet [5]. Dès lors, dans la pratique, les poids sont d'habitude limités à 4 , quitte à ce que des observations exceptionnelles soient éventuellement affectées de poids plus grands.

Nous n'avons pas ici à nous attarder sur les aspects numériques du problème général considéré, dont la résolution est fort longue, encore qu'aujourd'hui l'utilisation de l'ordinateur électronique en facilite le traitement. 


\section{Comment parvenir à calculer l'orbite d'une étoile double visuelle apparemment impossible à déterminer par la méthode de Thiele-Innes?}

Sous ce titre, l'occasion nous a été donnée de publier, en collaboration avec notre collègue brésilien de Freitas Mourao [6], un travail où apparaît, à plusieurs occasions, l'intérêt de la connaissance des erreurs d'observation.

Ainsi qu'on le sait, la méthode de Thiele-Innes relative au calcul d'orbites elliptiques d'étoiles doubles visuelles est basée sur la considération, dans l'orbite apparente relative, d'une part, de 3 lieux moyens $\left(t_{1}, \theta_{1}, \varrho_{1}\right),\left(t_{2}, \theta_{2}, \varrho_{2}\right),\left(t_{3}, \theta_{3}, \varrho_{3}\right)$ où les $\theta_{i}$ et $\varrho_{i}$ caractérisent, en coordonnées polaires, les positions du compagnon $B$ par rapport à l'étoile principale $A$, à des instants $t_{i}$ et, d'autre part, de la constante des aires.

Trois conditions doivent être remplies pour que cette méthode conduise à une solution. Les instants $t_{i}$ étant exacts, les seules données susceptibles d'être entachées d'erreur sont les $\theta_{i}, \varrho_{i}$ et la constante des aires constituant en tout 7 paramètres. Pour les besoins du but poursuivi ici, contentons-nous de considérer l'une des conditions, celle relative à l'excentricité orbitale $e$ : vu qu'il s'agit d'une ellipse, on doit avoir $e<1$. $\mathrm{Si}$, lors des calculs, on aboutit à une valeur de l'excentricité supérieure à l'unité, la question se pose de chercher à modifier les 7 paramètres de base dans des limites plausibles afin d'obtenir une correction suffisante pour ramener l'excentricité à être inféricure à l'unité. En différentiant la fonction $e=F\left(C, \varrho_{i}, \theta_{i}\right)$ il vient

$$
\mathrm{d} e={ }_{\partial C}^{\partial e} \mathrm{~d} C+\sum_{\partial \varrho_{i}}^{\partial e} \mathrm{~d} \varrho_{i}^{\prime \prime}+\sum_{R}^{1} \frac{\partial e}{\partial \theta_{i}} \mathrm{~d} \theta_{i}^{0}, \quad i=1,2,3,
$$

où $1 / R=0.01745$.

Cette relation montre notamment qu'en changeant les $\varrho_{i}$ de $\mathrm{d} \varrho_{i}^{\prime \prime}$ (en secondes d'arc) et les $\theta_{i}$ de $\mathrm{d} \theta_{i}^{\circ}$ (en degrés), il est possible d'atteindre le but poursuivi. L'expérience révèle que les dites corrections à apporter aux lieux fondamentaux sont de loin inférieures aux marges d'erreurs dont ces lieux sont susceptibles d'être entachés [7] et dont il a été question ci-avant (précision des mesures).

\section{Bibliographie}

[1] Arend, S.: 1949, 'Mesures micrométriques d'étoiles doubles', Ann. Obs. Roy. Belg., 3e série, IV, 147.

[2] Arend, S.: 1936, 'Sur le rattachement d'un astre à un nombre quelconque d'étoiles repères par la méthode des masses coordonnées', Bull. Soc. Belge Photogrammétrie n ${ }^{\circ}$ 8, déc., 5-14.

[3] Wierzbiński, St.: 1952, 'Orbite corrigée de ADS $8336=\beta 794$ ', Acta Astron., Cracovie, Série C, 5, 18-24.

[4] Karmel, G.: 1939, 'Definitive Bahnbestimmung des Doppelsternes $\Sigma 1037=$ ADS 5871', Astron Nachr. 269, 312-322.

[5] Volet, Ch.: 1931, 'Méthode pour le calcul des orbites d'étoiles doubles visuelles', Bull. Astron. 2e série, 7, 13.

[6] Arend, S. et de Freitas Mourao, R. R.: 1968, 'Comment parvenir à calculer l'orbite d'une étoile double visuelle apparemment impossible à déterminer par la méthode de Thiele-Innes?', Comm. Obs. Roy. Belg., Série A, n 5 .

[7] Arend, S. et de Freitas Mourao, R. R.: 1968, 'Étude complète de l'orbite elliptique de l'étoile double visuelle h $3447=$ BDS 836 ( $\tau$ Sculptoris)', Comm. Obs. Roy. Belg., Série A, n 6. 


\section{Discussion}

Couteau: Ne doit-on pas faire plusieurs itérations?

Arend: En général, ce n'est pas nécessaire. Les éléments sont liés. Vous ne pouvez changer les 7 paramètres que dans des limites restreintes possibles parce que toute l'orbite dépend des donnés, mais si vous changez les données d'une manière intempestive, même à votre insu, il est possible que l'orbite ne marche plus. Alors il faut raisonner de nouveau, voir ce qu'il convient de faire et agir comme il faut (dans le bon sens et de petites quantités) sur les 7 paramètres pour corriger l'excentricité.

Couteau: N'est-il pas judicieux de faire la contre épreuve par deux méthodes, par exemple: ThieleInnes et Danjon?

Arend: La méthode de Thiele-Innes est appliquée à Uccle. Le problème à résoudre ici est d'obtenir une orbite lorsqu'on y arrive pas. Ces formules-ci résolvent le problème. Mais l'orbite n'est pas bien déterminée. Il est intéressant de considérer toutes sortes de méthodes de détermination d'orbite. Mais il y a une chose mise en évidence par cette méthode, c'est que plus l'arc est grand, plus le résultat est meilleur.

Deutsch: Je me rappelle que Van Biesbroeck dans un article publié à 'Pacific' a conseillé de ne plus publier d'orbites préliminaires.

Heintz: One might avoid the uncertainty of the areal constant in the van den Bos method by determining the excentricity graphically first and, then work out the elements backward. I should prefer, in such cases, to use the Rabe method.

Duruy: La moyenne d'un ensemble de mesures n'est pas la valeur la plus probable de la quantité à mesurer que si:

(1) le nombre de mesures est grand,

(2) la courbe de fréquence est symétrique.

On reconnait cette symétrie précisément par le grand nombre de mesures. Ne pourrait-on pour les couples les plus intéressants (certaines orbitales) réserver un grand nombre de nuits par saison? Souvent on se borne à trois, ce qui est insuffisant pour justifier une valeur probable.

Arend: En principe, il faudrait un grand nombre d'observations et une répartition gaussienne; ce n'est pas le cas, mais il faut partir avec quelque chose et l'on s'en sert comme on peut; on arrive ainsi à faire des travaux comme ceux que je viens d'esquisser dans mon exposé; en ce qui concerne le nombre des observations, l'Union Astronomique Internationale incite à en faire pour certains couples déterminés. Le role de la circulaire d'information est de signaler les couples à observer. 